\title{
SEGMENTAL DILATATION OF THE SMALL INTESTINE
}

\author{
BY \\ STIG SJÖLIN and LARS THORÉN \\ From the Departments of Paediatrics and Surgery, University Hospital, Uppsala, Sweden
}

(RECEIVED FOR PUBLICATION APRIL 2, 1962)

In 1959 Swenson and Rathauser described three patients with an unusual dilatation of the colon. Each case had been referred to the hospital with a diagnosis of Hirschsprung's disease (congenital aganglionic megacolon), and on the whole the histories were consistent with this diagnosis. Histological examination of a pre-operative rectal biopsy specimen and a specimen taken during operation, however, demonstrated the presence of ganglion cells. In all three patients the dilated segment of colon was resected, and ganglion cells were also identified in the resected specimens. After the operation the patients were symptom free.

Recently we have seen a girl with a malformation very similar to that described by Swenson and Rathauser, but located to the small intestine, and with iron deficiency anaemia as the presenting sign.

\section{Case Report}

K.L., a female, was born June 7, 1955. At the age of 3 years she was admitted because of an acute upper respiratory infection and pallor which had become increasingly obvious during the last two months. Physical examination revealed an extreme pallor and a soft systolic murmur over the heart, but otherwise nothing abnormal. The haemoglobin concentration was $4.8 \mathrm{~g} . / 100 \mathrm{ml}$., mean corpuscular volume (M.C.V.) $79 \mu^{3}$, mean corpuscular haemoglobin (M.C.H.) $20 \mu \mu \mathrm{g}$., mean corpuscular haemoglobin concentration (M.C.H.C.) $25 \%$. The reticulocyte count was $2 \%$. The serum iron was $13 \mu \mathrm{g}$. $/ 100 \mathrm{ml}$., the total iron-binding capacity was $336 \mu \mathrm{g} . / 100 \mathrm{ml}$., but rose to $532 \mu \mathrm{g}$. $/ 100 \mathrm{ml}$. within a few days when the acute infection had been cured. The concentration of free erythrocyte protoporphyrins was very high (304 $\mu \mathrm{g} . / 100 \mathrm{ml}$.). Platelet and leucocyte counts were normal, as was the serum bilirubin concentration. These findings suggested the diagnosis of iron deficiency anaemia. Because the girl's diet seemed to have been adequate, her anaemia was probably due to chronic bleeding. However, we were not able to find any bleeding site. Repeated examination of the stools for occult blood was negative. Radiological examina- tion of the gastro-intestinal tract revealed no abnormality. Iron treatment, started a week after admission, resulted in a peak reticulocyte count of $22 \%$ and a rapid rise in haemoglobin.

During the following eight months she was seen regularly in the out-patient department. Her general state was good. The haemoglobin concentration varied between 10 and $12 \mathrm{~g} . / 100 \mathrm{ml}$. and the reticulocyte count between 2 and $12 \%$. There were no other signs of hyperhaemolysis. Bleeding from the gastro-intestinal tract was not noticed. Periodically she was treated with iron.

At the age of 3 years and 8 months she was readmitted to the hospital because her haemoglobin concentration had again fallen to low values (about $7 \cdot 5 \mathrm{~g}$./100 ml.). The haematological findings at this time were also consistent with an iron deficiency anaemia. Two positive examinations out of 14 for occult blood in the stools prompted us to repeat the radiological examination of the gastro-intestinal tract. Now a large sac-like widening of the small intestine could be seen (Fig. 1). The findings seemed to be best explained by a dilatation of the ileum proximal to an aganglionic segment, but alternative explanations, e.g. a duplication or an enormous diverticulum, were discussed.

Operation (L. Thorén): About $40 \mathrm{~cm}$. from Bauhin's valve the small intestine was markedly dilated (Fig. 2). The dilated segment was about $20 \mathrm{~cm}$. long, and the largest diameter was $8 \mathrm{~cm}$. In the middle of the dilated area was a one-inch long Meckel's diverticulum. Distal to the dilated portion was a small area which was slightly narrower and which then led into normal ileum. Proximal to the Meckel's diverticulum there was a circular scar with injected vessels, possibly indicating ulcerations on the inner side. The abdominal viscera were otherwise macroscopically normal.

The dilated segment together with $10 \mathrm{~cm}$. of normal gut distal and proximal to it were extirpated and an endto-end anastomosis of the small intestine was made. There were no post-operative complications.

Pathology. The dilated portion was cut open, and it was found to continue with normal intestinal mucous 


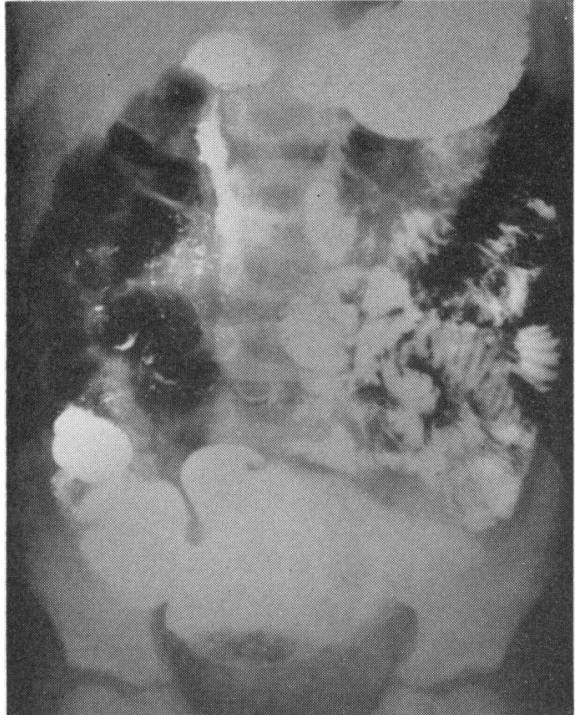

FIG. 1.- Radiograph showing the dilated segment in the distal ileum.

membrane proximally and distally without a definite boundary. The wall in the dilated area was rather thin but the mucous membrane appeared normal. Proximal to the Meckel's diverticulum was a long ulcer which encircled the intestine and was 7-8 $\mathrm{mm}$. wide.

Microscopic examination showed moderate inflam-

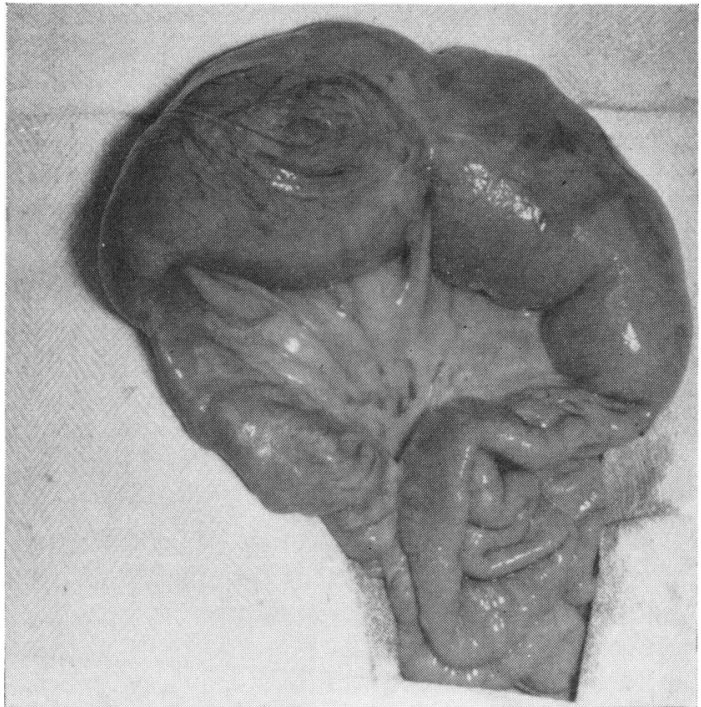

FIG. 2.-The dilated segment of the small intestine demonstrated at laparotomy. The small intestine on both sides of the dilatation with normal appearance.

matory cell infiltration, partly of plasma cells, in the submucosa. The muscle layer and the serosa were normal. In the distal section of the gut there was a normal muscle layer with abundant ganglion cells and nerve fibres (Fig. 3). The nerve tissues in the proximal section and from the dilated segment also seemed to be

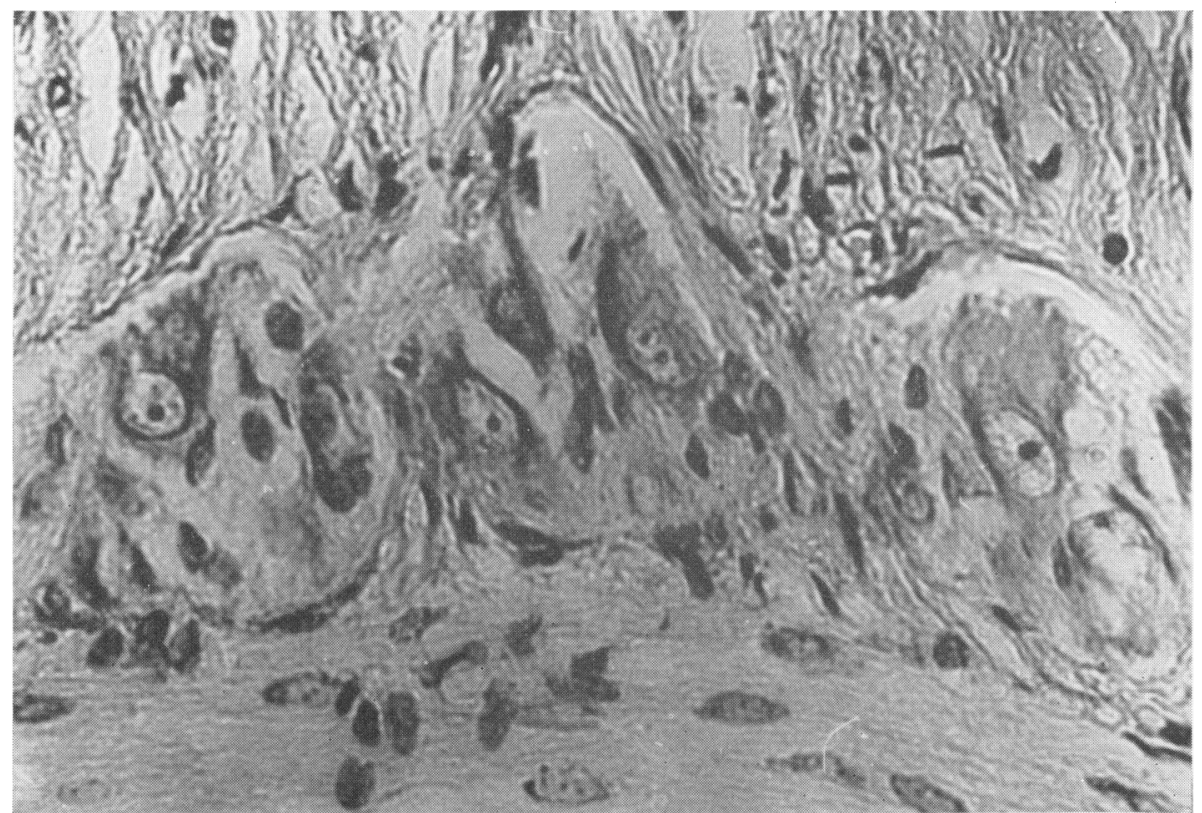

Fig. 3.-Microscopic section from the distal part of the specimen. An abundance of ganglion cells in both plexus myentericus and submucosus is demonstrated. The same picture was obtained in sections from other parts of the specimen. 
normal when stained by the methods of van Gieson and Nissl. Ganglion cells corresponding to both the myenteric and the submucous plexuses were demonstrated.

The gut thus showed a picture of a non-specific enteritis with lymphoid hyperplasia. The base of the circular ulcer showed a granulocyte infiltration and a non-specific inflammatory cell reaction. No atopic gastric mucous membrane could be found either in the small intestine or in Meckel's diverticulum.

The last follow-up examination was performed three years after operation, and she was found to be quite healthy with a normal blood picture and normal radiological findings in the small intestine.

\section{Discussion}

In this case the clinical picture is noteworthy mainly because the dilatation of the small intestine did not give rise to any proper abdominal symptoms. The case also emphasizes the importance of always regarding iron deficiency anaemia as only a symptom, the cause of which must always be made clear. The value of repeated examinations for occult blood in the stools in order to demonstrate intermittent or chronic bleeding from the alimentary tract is also well demonstrated, since only two of 21 investigations for blood in the faeces were positive. It also seems worth noting that a radiological investigation can give a normal picture even in the presence of considerable anatomical changes in the small intestine. If there is good reason to suspect a source of bleeding in the alimentary tract, repeated radio- logical investigations are clearly desirable and in some cases exploratory laparotomy. In children an ulcer in a Meckel's diverticulum is the commonest finding.

The pathological-anatomical picture in this case gives no basis for an aganglionic deformity. Ganglion cells were seen in both the myenteric plexus and the submucous plexus, and no changes could be observed in these with different stains. It can therefore be assumed that this is a developmental anomaly with segmental dilatation of the small intestine. It was not possible to demonstrate ectopic gastric mucosa in the Meckel's diverticulum as cause of the transverse ulcer which was the source of the bleeding.

\section{Summary}

A malformation appearing as a segmental dilatation of the small intestine of the ileum without aplasia of the ganglion cells is described in a girl who was operated on for this condition at the age of 4 years. The clinical picture was dominated by an iron-deficiency, haemorrhagic anaemia. On two occasions occult blood in the stools was found. Pre-operative radiographs showed a severe dilatation of a segment of small intestine. After this dilated segment of small intestine was resected the tendency to anaemia was eliminated.

\section{REFERENCE}

Swenson, O. and Rathauser, F. (1959). Segmental dilatation of the 